\title{
Highly Enantioselective Asymmetric Hydrogenation of $\beta$-Acetamido Dehydroamino Acid Derivatives Using a Three Hindered Quadrant Rhodium Catalyst
}

\author{
He-Ping Wu and Garrett Hoge*
}

\section{Supporting Information}

Materials and Methods. All reactions and manipulations were performed under nitrogen in standard laboratory glassware. Asymmetric hydrogenation was performed in a nitrogen filled glovebox. All anhydrous solvents used were obtained from Aldrich Chemical Co. and degassed by three freeze-pump-thaw prior to use. Hydrogen gas was used from a lecture bottle supplied by Specialty Gas. Hydrogenations were performed in a Griffin-Worden pressure vessel supplied by Kimble/Kontes. Substrates $\mathbf{3}$ and $\mathbf{5}$ were synthesized according to literature procedures. Thin-layer chromatography (TLC) was performed on precoated silica gel $60 \mathrm{~F}_{254} \mathrm{TLC}$ plates supplied by Merck KGaA. Flash chromatography was carried out by using silica gel 60 (particle size $0.063-0.200 \mathrm{~mm}$ ). $400 \mathrm{MHz}{ }^{1} \mathrm{H}$ NMR, $100 \mathrm{MHz}{ }^{13} \mathrm{C}$ NMR, and $162 \mathrm{MHz}{ }^{31} \mathrm{P}$ NMR spectra were obtained on a Varian Unity+400 (Inova400 after 08/15/00) spectrometer equipped with an Auto Switchable 4-Nuclei PFG probe, two RF channels, and a SMS-100 sample changer by Zymark. Mass Spectrometry was performed on a Micromass Platform LC system operating under MassLynx and OpenLynx open access software. Gas Chromatography (GC) was performed on a 110 volt Varian Star 3400 equipped with an FID detector with electrometer, a model 1061 packed/530 micron ID flash injector, a model 1077 split/splitless capillary injector, a relay board that monitors four external events, and an inboard printer/plotter.

General procedure for asymmetric hydrogenation reactions. Catalyst (S)-2 (0.005 mmole) and substrate $(0.50 \mathrm{mmol})$ were dissolved in $2.5 \mathrm{~mL}$ of solvent in a GriffinWorden pressure vessel and was then sealed and pressurized to the appropriate pressure of $\mathrm{H}_{2}$. The mixture was VIGOROUSLY stirred with a Teflon coated magnet at $25{ }^{\circ} \mathrm{C}$ until $\mathrm{H}_{2}$ uptake was complete. The $\mathrm{H}_{2}$ pressure in the bottle was released. The reaction mixture was then analyzed via chiral GC to provide the enantiomeric excess. 
Enantiomeric Excess Determinations. Enantiomeric excesses were determined via chiral GC as follows:

- Methyl-3-acetamidobutanoate (4) $\left(25 \mathrm{~m}\right.$ CP Chirasil-Dex CB, $125^{\circ} \mathrm{C}$, isothermal, $(\mathrm{S}) \mathrm{t}_{1}=7.67 \mathrm{~min} ;(\mathrm{R}) \mathrm{t}_{2}=8.21 \mathrm{~min}$.

- Ethyl 3-acetamidohexanoate (6a) $\left(25 \mathrm{~m} \mathrm{CP}\right.$ Chirasil-Dex $\mathrm{CB}, 108^{\circ} \mathrm{C}$, isothermal, (S) $\mathrm{t}_{1}=43.86 \mathrm{~min} ;(\mathrm{R}) \mathrm{t}_{2}=44.97 \mathrm{~min}$.

- Ethyl 3-acetamido-5-methylhexanoate (6b) $\left(25 \mathrm{~m} \mathrm{CP}\right.$ Chirasil-Dex CB, $115{ }^{\circ} \mathrm{C}$, isothermal, $(\mathrm{S}) \mathrm{t}_{1}=67.01 \mathrm{~min} ;(\mathrm{R}) \mathrm{t}_{2}=69.07 \mathrm{~min}$.

- Methyl 3-acetamido-4,4-dimethylbutanoate (6c) (30m Chiraldex-gamma-TA, $135^{\circ} \mathrm{C}$, isothermal, $(\mathrm{R}) \mathrm{t}_{1}=9.19 \mathrm{~min} ;(\mathrm{S}) \mathrm{t}_{2}=9.78 \mathrm{~min}$.

- Ethyl 3-acetamido-3-phenylpropanoate (6d) (25m CP Chirasil-Dex CB, $145^{\circ} \mathrm{C}$, isothermal, $(R) t_{1}=45.55$ min; $(S) t_{2}=47.64$ min.

- Ethyl 3-acetamido-4-methylpentanoate (6e) (25m CP Chirasil-Dex CB, $125{ }^{\circ} \mathrm{C}$, isothermal, $(\mathrm{R}) \mathrm{t}_{1}=14.32 \mathrm{~min} ;(\mathrm{S}) \mathrm{t}_{2}=14.89 \mathrm{~min}$.

Absolute Configurations. Absolute configurations of the following products were assigned by comparison of the signs of optical rotation with those of literature values:

- Methyl 3-acetamidobutanoate (4) $[\alpha]_{\mathrm{D}}{ }^{20}=+21.4\left(\mathrm{c} \mathrm{1.4}, \mathrm{CHCl}_{3}\right){ }^{\mathrm{i}}$

- Ethyl 3-acetamidohexanoate (6a) $[\alpha]_{\mathrm{D}}{ }^{20}=+42.8\left(\mathrm{c} 1.86, \mathrm{CHCl}_{3}\right){ }^{\mathrm{i}}$

- Ethyl 3-acetamido-5-methylhexanoate (6b) $[\alpha]_{\mathrm{D}}{ }^{20}=+44.6\left(\mathrm{c} 1.56, \mathrm{CHCl}_{3}\right){ }^{\mathrm{i}}$

- Ethyl 3-acetamido-3-phenylpropanoate (6d) $[\alpha]_{\mathrm{D}}{ }^{20}=-40.5(\mathrm{c} 2.15, \mathrm{MeOH})$.

- Ethyl 3-acetamido-4-methylpentanoate $(6 \mathrm{e})[\alpha]_{\mathrm{D}}{ }^{20}=+52.8\left(\mathrm{c} 1.2, \mathrm{CHCl}_{3}\right){ }^{\mathrm{i}}$

Absolute configuration of the following product was assigned tentatively by analogy to compounds $\mathbf{6 a}, \mathbf{6 d}$, and $\mathbf{6 e}$ by comparison of the signs of optical rotation:

- Methyl 3-acetamido-4,4-dimethylbutanoate (6c) $[\alpha]_{\mathrm{D}}{ }^{20}=+4.86$ (c 0.93 , $\mathrm{MeOH})$.

\footnotetext{
i Zhu, G.; Chen, Z.; Zhang, X. J. Org. Chem. 1999, 64, 6907-6910.

${ }^{\text {ii }}$ Zhou, Y.-G.; Tang, W.; Wang, W.-B.; Li, W.; Zhang, X. J. Am. Chem. Soc. 2002, 124, 4952-4953.
} 